\title{
Patrón de actividad diaria y de temperaturas ambientales y microambientales en una población de la lagartija endémica colombiana Anolis huilae (Squamata, Dactyloidae)
}

\author{
기 David Alfonso Bejarano-Bonilla*, \) Manuel Hernando Bernal-Bautista
}

Grupo de Herpetología, Ecofisiología y Etología, Universidad del Tolima, Ibagué, Colombia

\begin{abstract}
Resumen
La determinación del patrón de actividad de una especie, especialmente si es endémica o está amenazada, es valiosa para un programa de conservación efectivo. En este estudio se reporta el patrón de actividad diaria de una población de Anolis huilae y su relación con las temperaturas ambientales y microambientales. El trabajo se llevó a cabo con una población del municipio de Ibagué, Colombia, durante el 2017 y en su curso se registraron el número de animales activos cada hora desde las 6:00 hasta las 18:00 horas, así como las temperaturas ambientales y microambientales. Se encontró que la actividad de A. huilae era mayor en las horas del mediodía, entre las 12:00 y las 14:00 horas, cuando las temperaturas ambientales y microambientales son las más altas del día. Este resultado concuerda con el comportamiento de otras especies de Anolis, pero contradice otros estudios en lagartos en los cuales se reporta una menor actividad en las horas del mediodía. La diferencia entre los resultados mencionados podría atribuirse al hábitat de estas especies, ya que $A$. huilae vive principalmente en lugares boscosos protegidos de la radiación directa del sol, en tanto que las otras especies habitan principalmente áreas abiertas. Por lo tanto, es posible que la relación entre la cobertura del hábitat y la exposición a la radiación solar influya en el patrón de actividad diaria de los lagartos. (C) 2019. Acad. Colomb. Cienc. Ex. Fis. Nat.
\end{abstract}

Palabras clave: Anolis huilae; Comportamiento; Fisiología térmica; Lagartijas; Patrón de actividad; Temperatura.

Daily activity pattern and environmental and microenvironmental temperatures in a population of the Colombian endemic lizard Anolis huilae (Squamata, Dactyloidae)

\begin{abstract}
The identification of the activity pattern of a species, especially if it is endemic or threatened, is valuable information for an effective conservation program. This research reports the daily activity pattern of a population of Anolis huilae and its relationship with the environmental and micro-environmental temperatures. The work was performed within a population of the municipality of Ibagué, Colombia, during 2017, in which the number of active animals was recorded every hour from 6:00 to 18:00 hours together with the environmental and micro-environmental temperatures. We found that $A$. huilae is more active at midday, between 12:00 and 14:00 hours, when the environmental and micro-environmental temperatures are the highest. This finding agrees with the behavior of some Anolis species but disagrees with other studies in lizards that report a lower activity at midday when the highest environmental temperatures are recorded. The difference between these results may be attributed to the habitat of the species since $A$. huilae lives in forest places protected from the direct radiation of the sun, while the other species live mainly in open areas. Therefore, it is possible that the relationship between habitat coverage and exposure to solar radiation influences the daily activity pattern in lizards. (C) 2019. Acad. Colomb. Cienc. Ex. Fis. Nat.
\end{abstract}

Key words: Activity pattern; Anolis huilae; Behavior; Lizards; Temperature; Thermal physiology.

\section{Introducción}

La actividad de un animal es un concepto amplio; sin embargo, en estudios herpetológicos está asociada a movimientos para escapar de los depredadores y buscar alimento, pareja reproductiva o sitios para regular su temperatura (Gibbons \& Semlitsch, 1987). Por otra parte, el intervalo de tiempo en que los ectotermos son activos depende del clima, la intensidad de la luz solar, la duración del fotoperiodo, la temperatura ambiental y el horario de actividad de sus presas (Díaz \& Cabezas-Díaz, 2004). Específicamente, las variaciones en la actividad temporal, sea diaria o estacional, así como en el uso del microhábitat, son fundamentales para

\footnotetext{
*Correspondencia:

David Alfonso Bejarano Bonilla; trumandavid0101@gmail.com

Recibido: 3 de agosto de 2018

Aceptado: 11 de enero de 2019

Editor: Martha Patricia Ramírez Pinilla
} 
la termorregulación en ectotermos (Carretero \& Llorente, 1993; Hanato, et al., 2001), que es una característica fisiológica y de comportamiento fundamental para el desempeño óptimo de este grupo de animales.

Se considera que las especies ectotérmicas de hábitats boscosos tropicales son vulnerables al cambio climático, en especial a los incrementos de las temperaturas ambientales, ya que viven en áreas con mayor cobertura vegetal, no están adaptadas a las altas temperaturas de los ambientes abiertos y presentan pocas opciones de comportamiento para evadir las temperaturas altas (Tewksbury, et al., 2008). Además, el rango de temperatura para su desempeño óptimo es estrecho (Tewksbury, et al., 2008; Huey, et al., 2009; Sinervo, et al., 2010; Huey, et al., 2012), por lo que las alteraciones en la temperatura ambiental pueden conducir a la disminución de su eficacia biológica (Tewksbury, et al., 2008). En este contexto, el presente trabajo tuvo el propósito de aportar al conocimiento de la biología de la lagartija Anolis huilae Williams, 1982, especie endémica de Colombia. Como resultado, se reporta por primera vez su patrón de actividad diaria y la relación con las temperaturas ambientales y microambientales a las que se encuentra expuesta en su hábitat. Con dichos resultados se pretende establecer si estas lagartijas son más activas en las horas de mayor temperatura debido a su condición ectotérmica, pues a mayor temperatura sus procesos fisiológicos se aceleran, o si, por el contrario, son menos activas en las horas en que las temperaturas son más altas, ya que podrían experimentar una temperatura corporal mayor a su temperatura crítica máxima, como ocurre en otros lagartos (Sinervo, et al., 2010).

\section{Metodología}

Especie de estudio. Anolis huilae Williams, 1982 (Squamata: Dactyloidae) es una especie de lagartija endémica de la Cordillera Central de Colombia, localizada en los departamentos de Tolima y Huila, entre los 1.500 y los 1.750 m.s.n.m. (Williams, 1982), aunque en Ibagué se ha observado hasta a los 2.000 m.s.n.m. (obs. pers.). En la región del Tolima es conocida con el nombre común de "camaleoncito verde" y generalmente se encuentra perchada cabeza abajo en árboles o arbustos de matorrales entre los 1 y $7 \mathrm{~m}$ de altura (Williams, 1982). No se encuentra listada en ninguna categoría de amenaza según la Unión Internacional para la Conservación de la Naturaleza (UICN, 2017). Los machos son verdes con puntos claros y saco gular amarillo con líneas longitudinales formadas por pequeños puntos oscuros y una longitud de hocico a cloaca (LHC) de 70,5 mm ( \pm 5,6 mm). Las hembras, ligeramente más pequeñas, son de color café con dos polimorfismos, uno con manchas oscuras dorsales y otro con una línea dorsal beige, y presentan una LHC de 62,8 ( $\pm 5,4 \mathrm{~mm}$ ) (Figura 1). Según registros personales en el municipio de Ibagué, se han observado individuos copulando en los meses de enero, abril y septiembre, así como nidadas de 2 a 34 huevos.
Area de estudio. La investigación se desarrolló en el corregimiento de Juntas, municipio de Ibagué, departamento del Tolima, Colombia, en los predios del Ecolodge Ukuku

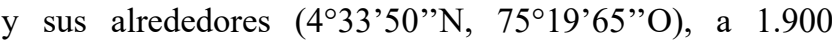
m.s.n.m., en una área aproximada de $40 \mathrm{Ha}$. La zona de estudio se caracteriza por tener parches de bosque secundario y matorrales surcados por caminos.

Patrón de actividad diaria. Para establecer la actividad diaria de la población de $A$. huilae en estudio, se seleccionaron tres transectos lineales contiguos de $500 \mathrm{~m}$ cada uno. A lo largo de siete días, entre febrero y marzo de 2017 , dos personas recorrieron cada transecto de manera alterna entre las 06:00 y las 18:00 horas, registrando cada hora el número de individuos observados para un total de 252 horas/hombre (pareja) de esfuerzo de muestreo (12 horas diarias x 3 transectos X 7 días). Cada pareja de observadores registró el número máximo de animales perchados o en movimiento en cada intervalo de muestreo, el cual se tomó como índice de la actividad diaria de las lagartijas (Logan, et al., 2015). Solo se registraron los datos de los individuos adultos, es decir, aquellos con una LHC mayor a $55 \mathrm{~mm}$ (Li, et al., 2011). Debido a que el estudio se realizó con lagartijas localizadas en transectos restringidos a los parches de bosque, es posible que un mismo animal se haya contado varias veces durante los siete días de muestreo, por lo que los resultados del presente trabajo se aplican específicamente a la población de estudio. No obstante, el número máximo de animales reportados sí correspondió al total de lagartijas observadas en cada hora, ya que fueron registradas simultáneamente por los tres grupos de trabajo en los tres diferentes transectos establecidos.

Registro de las temperaturas ambientales y microambientales. En el curso de los registros del patrón de actividad diaria de A. huilae, se tomó la temperatura
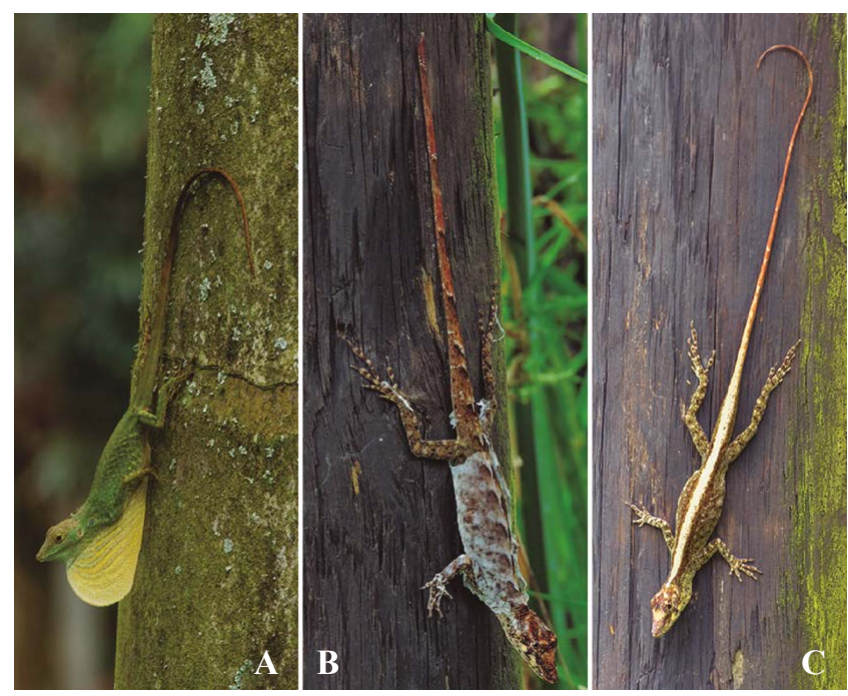

Figura 1. Individuos de Anolis huilae de Ibagué, Colombia. A. Macho en despliegue gular. B. Hembra con polimorfismo de manchas en muda. C. Hembra con polimorfismo de línea dorsal 
microambiental $\left(\mathrm{T}_{\text {micro }}\right)$ en el lugar donde se encontraron perchadas las lagartijas con ocho sensores de temperatura $\left(\right.$ iButton $^{\circledR}$ ) cada hora. Además, para medir la temperatura ambiental $\left(\mathrm{T}_{\mathrm{amb}}\right)$ se instaló un termohigrómetro EL-USB 2-LCD ${ }^{\circledR}$ en la parte exterior del Ecolodge Ukuku, área de confluencia de los tres transectos de estudio, suspendiéndolo bajo techo a una altura de 2,5 m del suelo para simular algo de sombra, y se lo programó para tomar registros cada hora.

Análisis de datos. Para establecer si hubo diferencias en la actividad diaria entre los machos y las hembras en cada periodo de muestreo de una hora, se hizo una prueba pareada de Wilcoxon, con la cual también se comparó el promedio de las temperaturas ambientales $\left(\mathrm{T}_{\mathrm{amb}}\right)$ con el de las temperaturas microambientales $\left(\mathrm{T}_{\text {micro }}\right)$ en cada hora del día. Por último, se utilizó la correlación de Spearman para establecer la asociación tanto de la temperatura ambiental como de la microambiental entre las 6:00 y las 18:00 horas con el número de animales activos durante el periodo de estudio. El análisis estadístico de los datos se hizo con los programas InfoStat, versión 2017, e IBM ${ }^{\circledR}$ SPSS $^{\circledR}$ Statistic, versión 21.0.0, tomando como valor de significación un $\mathrm{p}$ menor de 0,05 (Zar, 1996).

\section{Resultados}

No se encontraron diferencias significativas entre el número de hembras y machos activos a lo largo de cada una de las 12 horas de muestreo $(\mathrm{Z}=0,49 ; \mathrm{n}=12 ; \mathrm{p}=0,56)$. Al unir los datos de los machos y las hembras, se registró un mínimo de 15 y un máximo de 29 individuos para cada hora de muestro en los tres transectos de estudio (Tabla 1). Además, se observó que las lagartijas presentaron un patrón de actividad diaria unimodal, con un pico máximo de animales activos entre las 12:00 y 14:00 horas (Figura 2A).
Las temperaturas ambientales y microambientales mostraron el mismo patrón, con un pico máximo de $24,7^{\circ} \mathrm{C}$ para la temperatura ambiental entre las 12:00 y 13:00 horas, y de $21,5^{\circ} \mathrm{C}$ para las temperaturas microambientales entre las 14:00 y 16:00 horas (Figura 2B). Las temperaturas microambientales fueron menores que las ambientales (Tabla 1), con diferencias significativas $(Z=-2,59 ; n=12 ; p=0,005)$, y las lagartijas fueron más numerosas en las temperaturas altas ambientales $(\mathrm{r}=0,958 ; \mathrm{n}=13 ; \mathrm{p}<0,05)$ y microambientales $\left(r_{s}=0,742 ; n=12 ; p<0,05\right)$ (Figura 3).

\section{Discusión}

Según los resultados del estudio, A. huilae presentó un patrón de actividad diaria unimodal con picos máximos alrededor del mediodía (entre las 12:00 y las 14:00 horas), cuando las temperaturas ambiental y microambiental eran las más altas (Figura 2). Este patrón de actividad es similar al encontrado en otras especies del mismo género, como A. cristatellus (Huey, 1983), A. fuscoauratus (Vitt, et al., 2003), A. nitens (Vitt, et al., 2008), A. onca (Velásquez, et al., 2011) y $A$. heterodermus (Méndez-Galeano \& Calderón-Espinosa, 2017), las cuales presentan una mayor actividad entre las 11:00 y las 13:00 horas. En consecuencia, estos resultados concuerdan con la hipótesis de que la actividad de las lagartijas está relacionada con las temperaturas ambientales y corporales (Hanato, et al., 2001) y que la variación de la actividad de los lagartos diurnos está determinada por el ambiente y la fisiología termal de la especie (Adolph \& Porter, 1993), aunque difieren de lo señalado por Sinervo, et al. (2010), quienes mencionan que la actividad de los animales decrece cuando las temperaturas son mayores. La diferencia entre estos resultados puede atribuirse a que los lagartos estudiados por estos autores habitan zonas

Tabla 1. Temperaturas registradas y número de individuos observados durante el estudio del patrón de actividad diaria de Anolis huilae en Ibagué, Colombia

\begin{tabular}{|c|c|c|c|c|c|}
\hline Hora & $\overline{\mathbf{x}} \mathrm{T}_{\mathrm{amb}} \pm \mathrm{DE}\left(\left(^{\circ} \mathrm{C}\right)\right.$ & $\overline{\mathbf{X}} \mathbf{T}_{\text {micro }} \pm \mathrm{DE}\left({ }^{\circ} \mathrm{C}\right)$ & No. Max. ++ & No. Max. $0^{\lambda}$ & $\mathbf{n}$ \\
\hline $6: 00-7: 00$ & $15,1 \pm 0,2$ & $15,0 \pm 1,2$ & 8 & 7 & 15 \\
\hline 7:00-8:00 & $16,9 \pm 0,5$ & $15,6 \pm 1,3$ & 8 & 8 & 16 \\
\hline $8: 00-9: 00$ & $19,7 \pm 1,2$ & $17,0 \pm 1,8$ & 10 & 9 & 19 \\
\hline 9:00-10:00 & $22,4 \pm 1,3$ & $18,6 \pm 2,0$ & 12 & 12 & 24 \\
\hline 10:00-11:00 & $23,4 \pm 1,2$ & $19,9 \pm 2,0$ & 14 & 13 & 27 \\
\hline $11: 00-12: 00$ & $23,9 \pm 1,2$ & $20,7 \pm 2,0$ & 15 & 14 & 29 \\
\hline $12: 00-13: 00$ & $24,6 \pm 1,1$ & $21,2 \pm 2,2$ & 14 & 14 & 28 \\
\hline 13:00-14:00 & $23,8 \pm 0,9$ & $21,5 \pm 2,6$ & 14 & 15 & 29 \\
\hline $14: 00-15: 00$ & $22,6 \pm 0,7$ & $21,5 \pm 2,7$ & 13 & 14 & 27 \\
\hline $15: 00-16: 00$ & $21,5 \pm 0,6$ & $21,1 \pm 1,9$ & 13 & 13 & 26 \\
\hline $16: 00-17: 00$ & $20,1 \pm 0,6$ & $20,3 \pm 2,2$ & 11 & 11 & 22 \\
\hline 17:00-18:00 & $18,8 \pm 0,8$ & $19,4 \pm 2,0$ & 10 & 9 & 19 \\
\hline
\end{tabular}

$\overline{\mathrm{x}}$ : promedio; $\mathrm{DE}$ : desviación estándar; $\mathrm{T}_{\mathrm{amb}}$ : temperatura ambiental; $\mathrm{T}_{\text {micro }}$ : temperatura microambiental; No. Max.: número máximo de individuos registrados; $\hat{O}$ : machos; : $:$ hembras 

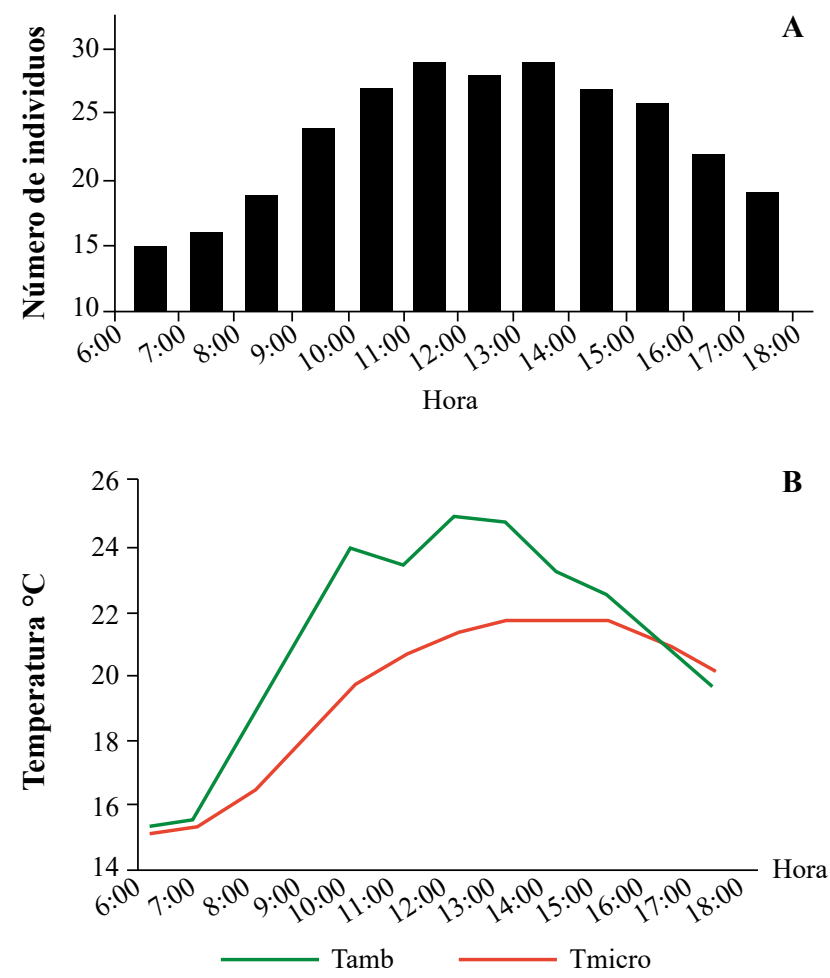

Figura 2. A. Patrón de actividad diario de Anolis huilae de Ibagué, Colombia. B. Promedio de las temperaturas ambientales $\left(\mathrm{T}_{\mathrm{amb}}\right)$ y microambientales $\left(\mathrm{T}_{\text {micro }}\right)$ registradas durante el estudio
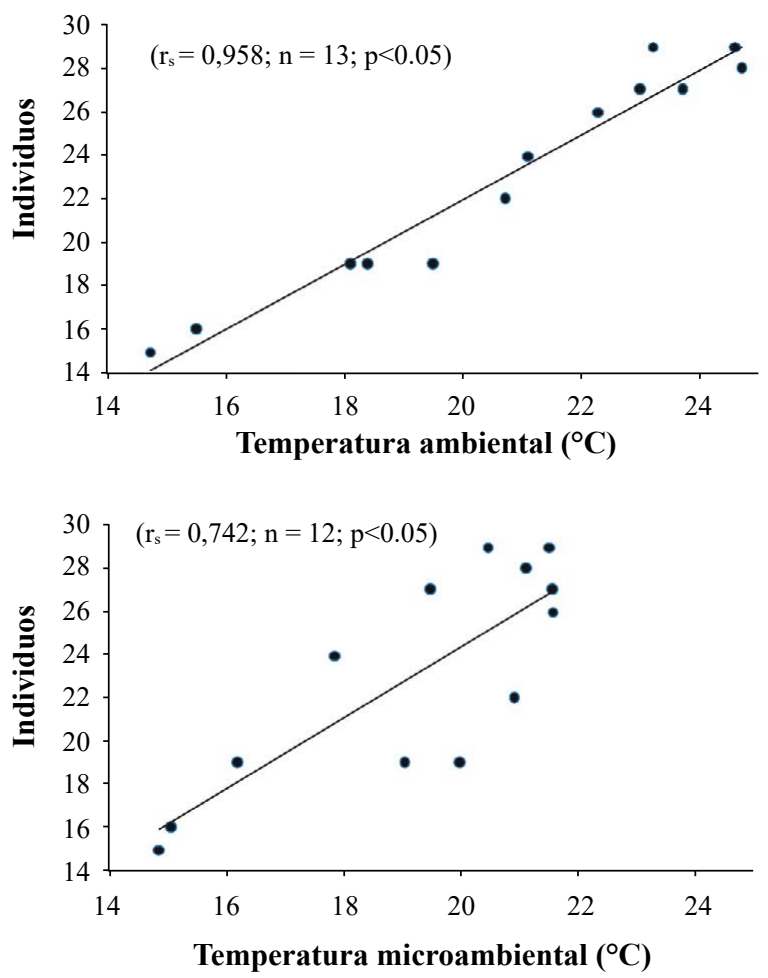

Figura 3. Correlaciones lineales entre el número de individuos activos de Anolis huilae y las temperaturas ambientales y microambientales en Ibagué, Colombia abiertas y están expuestos a temperaturas ambientales que pueden superar sus límites térmicos críticos máximos $\left(\mathrm{CT}_{\max }\right)$, por lo que los animales se refugian durante las horas de mayor temperatura, en tanto que $A$. huilae habita zonas boscosas, sombreadas, y se localiza principalmente en perchas que la protegen de la exposición directa de la radiación solar ofreciéndole temperaturas microambientales significativamente menores que las ambientales (Figura 2B). Probablemente la amortiguación térmica generada por el bosque o el matorral hace que estas lagartijas prefieran las temperaturas microambientales diarias más altas para maximizar su actividad funcional y su comportamiento, además porque, según los experimentos preliminares (datos sin publicar), dichas temperaturas son bastante inferiores a sus temperaturas críticas máximas.

Vitt, et al. (2008) plantean que las lagartijas de tamaños pequeños son termoconformistas y tigmotérmicas, ya que sus temperaturas corporales están supeditadas básicamente a la temperatura ambiental y a la del sustrato de percha. En este sentido, A. huilae podría ajustarse térmicamente a esta clasificación, ya que es un ecomorfo de tamaño pequeño del tipo trunk-ground (Huey, et al., 1983). Sin embargo, el uso de estas estrategias termorreguladoras debe validarse aplicando metodologías como la propuesta por Hertz, et al. (1993), la cual requiere recolectar datos sobre las preferencias térmicas y la sensibilidad térmica de algunas variables fisiológicas. Esta información es importante para determinar los posibles efectos antrópicos sobre la conservación de esta lagartija endémica de Colombia, especialmente por el actual cambio climático y la acelerada destrucción de los bosques que constituyen su hábitat, lo cual conlleva un incremento de las variables de su ambiente térmico que podría afectar su comportamiento termorregulatorio y su patrón de actividad diaria.

En el estudio se pudo observar que $A$. huilae es una especie de actividad diurna, es decir, durante las horas de luz, aproximadamente entre las 6:00 y las 18:00 horas. Sin embargo, algunas lagartijas descendieron de sus perchas al suelo alrededor de las 05:45 horas y finalizaron su actividad un poco después de las 18:00 horas. El inicio de la actividad de estas lagartijas en el amanecer podría deberse al ligero aumento de la temperatura ambiental, ya que según Pianka (1993), las especies del género Anolis, particularmente las del trópico, perciben con facilidad las variaciones térmicas ambientales. Este comportamiento también podría atribuirse a un ritmo circadiano endógeno de los reptiles que regula su actividad diaria (Firth \& Belan, 2007), o a una estrategia contra la depredación, ya que coincide con el inicio de la actividad de las aves, que son sus potenciales depredadores (Poulin, et al., 2001; Huang, et al., 2011; Marshal, et al., 2016). Además, el que la mayoría de los lagartos adultos sean activos en las horas del mediodía sugeriría que esta especie es especialista desde el punto de vista térmico, como lo indica Avery (1982) para algunas especies ectotermas que escogen un rango de temperatura 
estrecho para su actividad diaria, y Addo-Bediako, et al. (2000) y Cruz, et al. (2005), quienes mencionan que las especies con un estrecho rango de distribución altitudinal (como sucede con $A$. huilae) son generalmente especialistas termales. El que $A$. huilae pueda ser una especie de este tipo es otro dato importante para establecer el efecto de los cambios térmicos bruscos en su microhábitat (Huey, et al., 2009). También es posible que, al igual que otras especies del género Anolis (Muñoz, et al., 2014), A. huilae presente una gran plasticidad fisiológica y de comportamiento, en cuyo caso es igualmente necesario conocer hasta dónde llegan sus límites térmicos para enfrentar los desafíos impuestos por los cambios ambientales locales y globales.

\section{Conclusiones}

Anolis huilae presentó un patrón de actividad diario unimodal, con el mayor pico de actividad entre las 12:00 y las 14:00 horas, cuando las temperaturas ambientales y microambientales son las más altas. Esta lagartija habita lugares sombreados en los parches de bosques o en los matorrales, por lo que durante sus horas de actividad diaria está protegida de las temperaturas ambientales altas y de la radiación solar directa. Probablemente esta sea la razón de que presente una mayor actividad diaria hacia las horas del mediodía, con lo cual incrementa su temperatura corporal sin alcanzar su límite térmico crítico, a diferencia de otros lagartos que viven en áreas abiertas y permanecen inactivos en las horas de mayor temperatura diaria.

\section{Agradecimientos}

A la bióloga Annie M. Jiménez por su apoyo durante todo el estudio; a los estudiantes de Biología de la Universidad del Tolima Diego Valbuena, Diana Díaz Moreno, Luisa Fernanda Velásquez, Carlos Felipe Hernández y James Herrán por su colaboración en campo; a los propietarios del Ecolodge Ukuku Rural Lodge por facilitar sus locaciones, y a la oficina de investigaciones de la Universidad del Tolima por el financiamiento parcial de la investigación en el marco del macroproyecto número 510114.

\section{Contribución de los autores}

Ambos autores contribuyeron al análisis de los datos y a la escritura del manuscrito.

\section{Conflicto de intereses}

Ninguno

\section{Referencias}

Addo-Bediako, A., Chown, S. L., Gaston, K. J. (2000). Thermal tolerance, climatic-variability and latitude. Proceeding of the Royal Society of London B. 267: 739-745.

Adolph, S. C., Porter, W. P. (1993). Temperature, activity, and lizard life histories. American Naturalist. 142: 273-295.

Avery, R. A. (1982). Field studies of body temperatures and thermoregulation. En C. Gans and F. H. Pough (Eds). Biology of the Reptilia. Vol. 12. Physiology C. Physiological Ecology, p. 93-166. New York, USA: Academic Press.
Carretero, M., Llorente, G. (1993). Ecología térmica y actividad de una población costera de Psammodromus hispanicus. Revista Española de Herpetología. 7: 21-32.

Cruz, F. B., Fitzgerald, L. A., Espinoza, R. E., Schulte II, J. A. (2005). The importance of phylogenetic scale in test of Bergmann's and Rapoport's rules: lessons from a clade of South American lizards. Journal of Evolutionary Biology. 18: 1559-1574.

Díaz, J., Cabezas-Díaz, S. (2004). Seasonal variation in the contribution of different behavioural mechanism to lizard thermoregulation. Functional Ecology. 18: 867-875.

Firth, B.T., Belan, I. (2007). Daily and seasonal rhythms in selected body temperature in the Australian lizard Tiliqua rugosa (Scincidae): field and laboratory observations. Physiological Zoology. 71: 303-311.

Gibbons, J. W., Semlitsch, R. D. (1987). Activity patterns. En Seigel, R. A., Collin, J. T. and Novak, S. S. (Eds). Snakes: Ecology and Evolutionary Biology. p. 396-421. New York, USA: McMillan Publishing Company.

Hanato, F., Vrcibradic, D., Galdino, C., Cunha-Barros, M., Rocha, C., Van Sluy, M. (2001). Thermal ecology and activity patterns of the lizards community of the Restinga of Jurabatiba, Macaé, R. J. Revista Brasileira de Biologia. 61: 287-294.

Hertz, P. E., Huey, R., Stevenson, R. D. (1993). Evaluating temperature regulation by field-active ectotherms: the fallacy of the inappropriate question. American Naturalist. 142: 796-818.

Huey, R. B. (1983). Natural variation in body temperature and physiological performance in a lizard (Anolis cristatellus). En Rhodin, A. G. and Miyata, K. (Eds). Advances in Herpetology and Evolutionary Biology. p. 484-490. Cambridge, Massachusetts, USA: Museum of Comparative Zoology.

Huey, R. B., Deutsch, C. A., Tewksbury, J. J., Vitt, L. J., Hertz, P. E., Pérez, H. J. A. (2009). Why tropical forest lizards are vulnerable to climate warming. Proceedings of the Royal Society B-Biological Sciences. 276: 1939-1948.

Huey, R. B., Kearney, M. R., Krockenberger, A., Holtum, J. A. M., Jess, M., Williams, S. E. (2012). Predicting organismal vulnerability to climate warming: roles of behaviour, physiology and adaptation. Philosophical Transactions of the Royal Society B-Biological Sciences. 367: 1665-1679.

Huey, R. B., Pianka, E. R., Schoener, T. W. (1983). Lizard Ecology, Studies of a Model Organism. Massachusets, USA: Harvard University Press.

Huang, B., Lubarsky, K., Teng, T., Blumstein, D.T. (2011). Take only pictures, leave only...fear? The effects of photography on the West Indian anole Anolis cristatellus. Current Zoology 57: 77-82.

IUCN. (2017). IUCN Red List of Threatened Species. Version 2017.2. Fecha de consulta: abril de 2017. Disponible en: www.iucnredlist.org.

Li, H., Qu, Y. F., Ding, G. H., Ji, X. (2011). Life-history variation with respect to experienced thermal environment in the lizard, Eremias multiocellata (Lacertidae). Zoological Science. 28: 332-338.

Logan, M.L., Fernández, S.G., Calsbeek, R. (2015). Abiotic constraints on the activity of tropical lizards. Functional Ecology. 29: 694-700. 
Marshal, K. L. A., Philpot, K. E, Stevens, M. (2016). Microhabitat choice in island lizards enhances camouflage against avian predators. Scientific Reports. 6: 1-10.

Muñoz, M. M., Stimola, M. A., Algar, A. C., Conover, A., Rodríguez, A. J., Landestoy, M. A., Bakken, G. S., Losos, J. B. (2014). Evolutionary stasis and lability in thermal physiology in a group of tropical lizards. Proceedings of the Royal Society B. 281: 2013-2433.

Méndez-Galeano, M. A., Calderón-Espinosa, M. L. (2017). Thermoregulation in the Andean lizard Anolis heterodermus (Squamata: Dactyloidae) at high elevation in the Eastern Cordillera of Colombia. Iheringia Serie Zoológica.107: 1-8.

Pianka, E. (1993). The many dimensions of a lizard ecological niche. En Valakos, E., Bohme, W., Pérez-Mellado, V. and Maragou, P. (Eds). Lacertids of the Mediterranean, A biological approach. p. 121-154. Athens, Bonn, Alicante: Hellenic Zoological Society.

Poulin, B., Lefebvre, G., Ibáñez, R. Jaramillo, C., Hernández, C., Rand, S. (2001). Avian predation upon lizards and frogs in a neotropical forest understorey. Journal of Tropical Ecology. 17: 21-40.

Sinervo, B., Méndez de la Cruz, F., Miles, D. B., Heulin, B., Bastiaans, E., Villagrán-Santa Cruz, M., Lara-Resendiz, R., Martínez-Méndez, N., Calderón-Espinosa, M. L., Mesa-Lázaro, R. N., Gadsden, H., Ávila, L.J., Morando, M., De la Riva, I. J., Sepúlveda, P. V., Duarte-Rocha, C.
F., Ibanguengoytía, N., Aguilar-Puntriano, C., Massot, M., Lepetz, V., Oksanen, T. A., Chapple, D. G., Bauer, A. M., Branch, W. R., Clobert, J., Sites Jr., J. W. (2010). Erosion of lizard diversity by climate change and altered thermal niches. Science. 328: 894-899.

Tewksbury, J. J., Huey, R. B., Deutsch, C. A. (2008). Putting the heat on tropical animals. Science. 320: 1296-1297.

Velásquez, J., González, L. A., Prieto-Arcas, A. (2011). Ecología térmica y patrón de actividad del lagarto Anolis onca (Squamata: Polycrotidae) en la península de Araya, Venezuela. Saber, Universidad de Oriente, Venezuela. 23 (1): 5-12.

Vitt, L., Avila-Pires, T., Zani, P., Sartorius S., Espósito, M. (2003). Life above ground: ecology of Anolis fuscoauratus in the Amazon rain forest, and comparisons with its nearest relatives. Canadian Journal of Zoology. 81: 142-156.

Vitt, L. J., Shepard, D. B., Vieira, G. H. C., Caldwell, J. P., Colli, G. R., Mesquita, D. O. (2008). Ecology of Anolis nitens brasiliensis in Cerrado woodland of Cantão. Copeia. 1: 144-153.

Williams, E. E. (1982). Three new species of the Anolis punctatus complex from Amazonian and inter-Andean Colombia, with comments on the eastern members of the Punctatus species group. Breviora. 467: 1-38.

Zar, J. H. (1996). Biostatistical Analysis. Upper Saddle River, New Jersey, USA: Prentice Hall. 